\title{
Evaluación en un curso de ecuaciones diferenciales con el apoyo de material en línea de matemáticas en contexto
}

\section{Socorro del Rivero-Jiménez Leonsio Ruiz-Moreno}

Instituto Tecnológico Superior de Cajeme

División de Ciencias Básicas, ITESCA

Carretera Internacional a Nogales km 2 s/n CP 85024,

Cd. Obregón, Sonora.

MÉXICO

\section{Abstract \\ (Evaluation in a Differential Equations Course Supported by Online Mathematics Material in Context)}

The purpose of this research is to evaluate, on the one hand, school achievement in a course of differential equations when contextualized teaching material is implemented in electrical circuits with the use of the Moodle platform as a support tool and on the other, to evaluate the students' perception regarding to the usefulness of said platform. The research is of mixed type (quantitative and qualitative), it is based theoretically and methodologically on Mathematics in the Context of Sciences. The quantitative evaluation is established through a comparative system among electronic engineering students: control group and experimental group. The results indicate that there is a relationship between the application of the teaching material with the use of the Moodle platform and obtaining improvements in the learning of differential equations in the context of electrical circuits. The qualitative evaluation is established through the focus group technique where the students gave their opinions on the Moodle, helps them to have a better understanding of the subject and develop autonomous learning.

Index terms: mathematical in context, first order differential equations, moodle, electrical circuits, and engineering.

\section{Introducción}

La problemática del aprendizaje de la matemática es de carácter mundial, se encuentra inmersa en cualquier nivel educativo y es bastante compleja ya que en ella participan múltiples factores que repercuten ya sea de manera directa o indirecta, tales como problemas personales, de tipo económico, didáctico, de carácter epistemológico, etc. Un síntoma identificado de esta problemática en el caso del nivel superior y en particular en las carreras del área de ingeniería, la cual nos ocupa en esta investigación, es el elevado índice de reprobación que presentan los estudiantes en pruebas objetivas.

Al respecto de esta problemática tan compleja, Camarena [1] comenta: 
A nivel mundial, es conocido el hecho del alto índice de reprobación en las asignaturas de matemáticas en áreas de ingeniería, la reprobación es sólo un síntoma de toda la problemática. En este conflicto inciden muchos factores de tipo social, económico, de orden curricular, asociados a la didáctica, que contribuyen en el aprendizaje y en la enseñanza de las matemáticas, relacionados a la formación de los docentes inferidos al propio tema de estudio, por causas de la infraestructura cognoscitiva de los alumnos.

Un factor que contribuye en la reprobación es el adquirir un aprendizaje deficiente a corto plazo, es decir no significativo, en la concepción de Ausubel [2]. Esto quiere decir que aprender significativamente es "Atribuir significado a lo que se debe aprender a partir de lo que ya se conoce", lo cual se puede deber a muchos factores como la presentación de los contenidos de un curso, la formación del docente, el conocimiento del alumno, la disposición que presente el alumno por aprender matemáticas etcétera.

El Instituto Tecnológico Superior de Cajeme (ITESCA) lugar donde se realizó esta investigación, se ubica en Ciudad Obregón, Sonora, México y ahí se ha presentado una problemática recurrente en las carreras del área de ingeniería, que es la falta de interés que presentan muchos estudiantes por aprender matemáticas, lo cual se debe, entre otras cosas, a que ésta se presenta generalmente descontextualizada de problemas prácticos de interés para el alumno; al respecto Camarena [3, p.1] afirma "Los estudiantes no tienen claro porque estudiar matemáticas y esto demerita la motivación a esta ciencia".

Existen algunos investigadores que han trabajado en relación a la problemática de desvinculación de la matemática con las áreas de estudio del alumno, entre ellos se puede mencionar a Camarena [1], [4], Muro [5], Olazábal [6], Trejo [7], De Pavia [8], Sauza [9], Alvarado [10], Rojas [11], Accostupa [12], Neira [13], Camarena y Flores [14], quienes se fundamentan en la teoría denominada Matemática en el Contexto de las Ciencias (MCC). En el primer trabajo desarrollado bajo esta teoría la cual tiene por título "Diseño de un curso de ecuaciones Diferenciales en el contexto de los circuitos eléctricos" se describen experiencias de aprendizaje significativo para que los estudiantes construyan su conocimiento sobre las ecuaciones diferenciales ordinarias lineales en el contexto de los circuitos eléctricos [15].

Por otro lado, un auge importante que ha tenido el sistema educativo en el proceso de enseñanza y aprendizaje es la incorporación de las tecnologías de la información y comunicación (TIC), tales como: computadoras, software educativos, plataformas virtuales, etc., las cuales ofrecen ciertas ventajas respecto a la enseñanza presencialsin tecnología, en particular las plataformas virtuales permiten tener acceso a materiales didácticos, poder interactuar con otros estudiantes y con el maestro desde cualquier lugar y en cualquier momento del curso.

Meza, Garita y Villalobos [16], citados por Vilchez [17], proponen que los procesos de enseñanza y aprendizaje de la matemática asistida por computadora, deben basarse en los siguientes principios: a) El uso de la computadora en el proceso de enseñanza aprendizaje de la matemática debe enmarcarse en un planteamiento educativo; b) La computadora debe incorporarse en el proceso de enseñanza aprendizaje de la matemática sólo cuando sea más eficaz o más eficiente que otros medios; c) La incorporación de la computadora en el proceso de enseñanza y aprendizaje de la matemática permite aumentar la eficiencia y eficacia de algunas estrategias que el docente utilizaba antes de incorporar la computadora; y d) El empleo de la computadora en el proceso de enseñanza aprendizaje de la matemática permite diseñar algunas estrategias didácticas que no es posible desarrollar con otros medios.

En este último punto, radica uno de los factores más importantes que justifican la necesidad de utilizar software y materiales educativos computarizados para la enseñanza de la matemática. En relación a esto Kolman [18], citado en Vilchez [17], afirma que en la existencia de ambientes matemáticos apoyados con tecnología se favorece la motivación y la curiosidad intelectual del estudiante.

De esta manera considerando la desvinculación de la matemática con la profesión de estudio del alumno y la importancia del uso de las TIC como apoyo en la formación del estudiante de ingeniería, se aborda la siguiente investigación.

\section{Planteamiento del problema}

Para las instituciones educativas, en particular las de nivel superior, existen indicadores que miden el nivel de calidad de la enseñanza de la educación como: reprobación, aprobación, deserción, etc. En el ITESCA se manejan los siguientes indicadores: a) Índice de Eficiencia Terminal; b) Índice de Reprobación; c) Índice de Titulación; yd) Índice de Deserción (se pueden consultar en http://www.itesca.edu.mxlitescalmisionvision.asp).

En particular, el indicador de reprobación en el ITESCA establece mantener el índice de reprobación menor o igual al ciclo inmediato anterior, el cual en el año 2013 era de $16.3 \%$, esto es, porque el rendimiento académico de los estudiantes universitarios es un indicador fundamental en la calidad de la enseñanza, tal como lo deja ver La Commission on Higher Education MSA [19, p. 19], citado por [20, p. 394]. 
Según datos del Departamento de Registro Escolar del ITESCA el rendimiento académico de los estudiantes en el área de Ingeniería es bajo. En el periodo de agosto a diciembre del año 2012 los índices de reprobación fueron altos; por ejemplo, en Cálculo Diferencial fueron de $56 \%$ y de $65 \%$, en Cálculo Integral fueron de $47 \%$ y de $36 \%$, en Cálculo Vectorial fueron de $60 \%$ y $82 \%$ y en Álgebra lineal fueron de $66 \%$ y de $41 \%$, los cuales superan por mucho al $16.3 \%$ establecido anteriormente. Por lo anterior surge la necesidad de ser más eficientes en el proceso de enseñanza-aprendizaje de las matemáticas lo que propicia buscar nuevas estrategias de enseñanza diferentes a la enseñanza tradicional.

Este trabajo de investigación se enfoca en la problemática que presenta la materia de ecuaciones diferenciales, particularmente ecuaciones de primer orden, debido a la importancia de ésta en el currículo de las carreras del área de ingeniería y por presentar un alto índice de reprobación. Un curso tradicional de ecuaciones diferenciales ordinarias es común que se presente de la siguiente forma: se define una ecuación diferencial como una ecuación que contiene derivadas o diferenciales de una o más variables dependientes, respecto de una o más variables independientes. Se muestran ejemplos, se clasifican de acuerdo al orden y la linealidad, se especifica qué es una solución particular, qué es la solución general y se define un algoritmo para la solución, dependiendo de si es separable, exacta, lineal, etc. Donde prevalece una cantidad considerable de ecuaciones diferenciales que el alumno deberá resolver. Posteriormente, el maestro evalúa si el alumno es capaz de resolver las ecuaciones diferenciales de los diferentes tipos, con lo cual sólo mide habilidades de tipo procedimental. Cabe hacer la aclaración que es bueno que el alumno adquiera este tipo de habilidades pero no es suficiente para que en él se genere un aprendizaje significativo debido a que no le encuentra sentido del porqué está resolviendo estas ecuaciones.

Esta práctica tradicionalmente se ha llevado a cabo en los diferentes niveles educativos, al respecto Moreno y Azcárate [21, p. 266], refieren que:

El profesor considera que el estudiante aprende por imitación, que es asimismo un receptor pasivo del discurso del docente, y que en ningún momento el propio profesor pueda ni siquiera plantearse que en una misma clase pueda haber estudiantes con diferentes estilos de aprendizaje, susceptibles de ser motivados si la enseñanza se orientará a sus cualidades específicas del aprendizaje.

Otro aspecto que afecta considerablemente el aprendizaje de las ecuaciones diferenciales son los conocimientos previos necesarios para la materia, ya que estos son generalmente muy deficientes. La gran mayoría de los alumnos no comprenden el concepto de derivada como "razón de cambio", "velocidad" (deri- vada de la posición), etc., y presentan procedimientos algebraicos erróneos así como técnicas de integración inadecuadas, entre otros. Al respecto Cantoral [22, p. 6], expresa que:

El pensamiento matemático debe operarse sobre una red compleja de conceptos unos avanzados y otros más elementales. Quizá por eso los estudiantes no puedan entender lo que significa una ecuación diferencial al menos de que entiendan a un cierto nivel, que va más allá del solo manejo de las técnicas asociadas, otros conceptos matemáticos, como la diferencial, la integral, la variable, o incluso, el número, y además deben articularlos bajo diferentes contextos de representación, como formas gráficas, ordenamientos numéricos, representaciones analíticas, lenguaje natural o procesamiento icónico de la información.

Con base en la problemática planteada, es de particular interés trabajar bajo la teoría de la Matemática en el Contexto de las Ciencias, porque ha mostrado reunir condiciones suficientes para el desarrollo de aprendizajes significativos. Al respecto Camarena [3, p. 13] comenta: "El factor motivación en el estudiante se encuentra altamente estimulado a través de la matemática en contexto y su desempeño académico como futuro profesionista se incrementa, es decir, la transferencia del conocimiento se puede establecer sin tantos tropiezos". Combinada esta teoría con el uso de la tecnología como apoyo al aprendizaje, como el caso de la plataforma Moodle por las ventajas que ésta representa al propiciar un aprendizaje sociocultural, se contará con una didáctica no tradicional.

Por otro lado, el rendimiento académico de los estudiantes es un indicador clave para determinar si una institución está alcanzando sus objetivos educativos. De aquí, que sea esencial la existencia de un programa de evaluación para documentar el rendimiento académico de los estudiantes.

Para definir la pregunta y objetivo de la investigación se requiere definir el término "rendimiento académico", se hace la aclaración de que no existe una definición precisa para este término debido a la complejidad del mismo, ya que se puede medir tanto cualitativa como cuantitativamente y en este proceso evaluativo intervienen gran número de variables tales como las políticas institucionales, test creados por el maestro, criterios tomados en academias, etc., por lo que no hay una estandarización dentro del mismo instituto para medir el rendimiento académico en todas las áreas.

En esta investigación se toma la conceptualización que presenta Pérez, Ramón y Sánchez [23], Vélez y Roa [24], citados en Garbanzo [25, p. 60], quienes lo definen como "el valor atribuido al logro del estudiante en su desempeño académico, mediante las calificaciones obtenidas. Casi siempre involucra una evaluación cuantitativa del aprovechamiento 
del estudiante, ya sea satisfactorio o no, e implica deserción o éxito académico".

En [21, p. 46] se establece:

Las notas obtenidas, como un indicador que certifica el logro alcanzado, son un indicador preciso y accesible para valorar el rendimiento académico, si se asume que las notas reflejan los logros académicos en los diferentes componentes del aprendizaje, que incluyen aspectos personales, académicos y sociales.

Así, la pregunta de investigación cuantitativa: en el marco de la teoría de la Matemática en el Contexto de las Ciencias, ¿el uso de material didáctico contextualizado en circuitos eléctricos e implementados en plataforma Moodle de apoyo al curso de ecuaciones diferenciales, incrementa el rendimiento académico de los estudiantes?

De esta forma se define el objetivo general como: "Evaluar el rendimiento académico de los estudiantes en el curso de ecuaciones diferenciales cuando cuentan con el apoyo de material didáctico de matemáticas contextualizadas en circuitos eléctricos, implementado en la plataforma Moodle".

\section{Hipótesis}

$\mathrm{H} 0$ : No existe relación entre la aplicación del material didáctico con el uso de la plataforma Moodle y la obtención de mejoras en el aprendizaje de las ecuaciones diferenciales en el contexto de los circuitos eléctricos.

H1: Existe relación entre la aplicación del material didáctico con el uso de la plataforma Moodle y la obtención de mejoras en el aprendizaje de las ecuaciones diferenciales en el contexto de los circuitos eléctricos.

La pregunta de investigación cualitativa es: ¿Cuál es la percepción de los estudiantes respecto al desarrollo del curso en cuanto al acceso, la navegación, la motivación y el aprendizaje?

La importancia de trabajar con circuitos eléctricos se debe a que una gran cantidad de objetos de la vida cotidiana requieren de circuitos eléctricos para su funcionamiento, por ejemplo, la televisión, la cafetera eléctrica, la computadora, etc., y son elementos de estudio indispensables para la carrera de ingeniería electrónica.

\section{Marco Teórico \\ 3.1 La Matemática en el Contexto de las Ciencias}

Camarena, autora de la teoría, menciona que la Matemática en el Contexto de las Ciencias reflexiona acerca de la vinculación que debe existir entre la matemática y las ciencias que la requieren. Esta se fundamenta en los siguientes paradigmas: a) La matemática es una herramienta de apoyo y disciplina formativa; b) La matemática tiene una función específica en el nivel universitario; y c) Los conocimientos nacen integrados[1], [15], [26], [27], [28], [29], citados por Camarena [3].

La teoría contempla cinco fases que están presentes en el ambiente de aprendizaje: la curricular, la didáctica, la epistemológica, la docente y la cognitiva las cuales emergieron en el orden presentado en los años 1984, 1987, 1988, 1990 y 1992 (véase Fig.1).

En esta investigación se trabajó con la fase didáctica la cual posee una estrategia didáctica que se denomina Matemática en Contexto [30], se encarga de que el alumno vea una matemática vinculada con sus intereses, sin aplicaciones artificiales, con la notación que requerirá en su carrera de estudio, no árida, de tal forma que logre conocimientos estructurados y no fraccionados, que construya su propio conocimiento con amarres firmes, duraderos y no volátiles y se encuentre motivado para que su desempeño académico se incremente, de tal forma que se le desarrollen las habilidades para la transferencia del conocimiento [31].

La Matemática en Contexto contempla nueve etapas metodológicas, en las cuales, para su implementación el maestro selecciona equipos formados por tres estudiantes con una característica especial, uno de ellos deberá ser el líder académico, otro el líder emocional y el tercero el líder operativo.

Etapas de la Matemática en Contexto [3, p. 6]:

1. Identificar los eventos contextualizados. Análisis de textos de las demás asignaturas que cursa el estudiante para de-

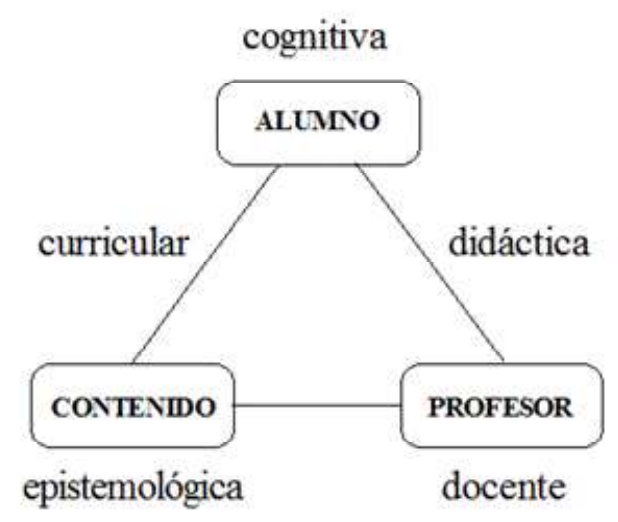

Fig. 1. Una terna dorada en educación [1]. 
terminar los eventos contextualizados que deberán ser planteados a los alumnos, siempre y cuando estén a su alcance cognitivo.

2. Plantear el evento contextualizado a los equipos.

3. Determinar las variables y las constantes del evento.

4. Incluir los temas y conceptos matemáticos y del contexto necesarios para el desarrollo del modelo matemático y solución del evento.

5. Determinar el modelo matemático.

6. Dar la solución matemática del modelo matemático.

7. Determinar la solución requerida por el evento.

8. Interpretar la solución en términos del evento y disciplinas del contexto.

9. Presentar una matemática descontextualizada. Recapitulación de los temas nuevos de matemáticas que han sido incorporados para la resolución del evento con el propósito de impartir una matemática descontextualizada, en donde se retoma la formalidad que sea necesaria según el área de estudio.

Las etapas 1, 2, 4 y 9 son actividades del docente, mientras que las etapas $3,5,6,7$ y 8 son actividades que desarrollan los estudiantes.

Aquí la etapa central es el modelaje matemático del evento contextualizado. Esta es la etapa en donde se pone de manifiesto la transferencia del conocimiento, entre las transferencias se encuentra la transferencia elemental que se refiere a la traducción del lenguaje natural al lenguaje matemático. Logrando esto, se considera que el estudiante ha sido capaz de vincular a las matemáticas con la disciplina del contexto.

La resolución de problemas es uno de los niveles de orden superior en las habilidades del pensamiento. Cuando son pro- blemas de matemáticas contextualizadas se requiere de los conocimientos disciplinarios previos para hacer uso de ellos en cualquier momento.

Cabe mencionar que la Matemática en Contexto se ha experimentado con asignaturas aisladas obteniéndose resultados prometedores. Como el trabajo de tesis de maestría de Hernández [32], "Las ecuaciones diferenciales ordinarias lineales de primer y segundo orden en el contexto del movimiento uniforme" donde se les presenta a los estudiantes situaciones problemáticas donde se resuelven problemas en el contexto de la Física con actividades didácticas de desplazamiento, velocidad y aceleración que se modelan con ecuaciones diferenciales lineales de primer y segundo orden. Y la investigación de Trejo, Camarena y Trejo [33], donde se trabaja con el caso particular de la construcción de un modelo matemático por medio de una ecuación diferencial para determinar matemáticamente el orden de una reacción química.

A partir de la teoría de la Matemática en el Contexto de las Ciencias, Camarena [34], diseña un Modelo Didáctico de Cursos en Línea.

\subsection{Modelo didáctico de cursos en línea}

El modelo didáctico utilizado en el presente trabajo es una adaptación de [34], el cual refiere al cambio de mentalidad en los actores del proceso educativo cuando es utilizada la educación en línea. Tanto el rol del estudiante como el del maestro, la comunicación entre ellos y los procesos didácticos tiene cambios en esta modalidad. Dado que el modelo didáctico está centrado en el aprendizaje, en los comandos principales y sus funciones, los roles de cada actor se describen en el cuadro 1, En [34]se expone: Uno de los aspectos importan-

Cuadro 1. Modelo centrado en el aprendizaje.

\begin{tabular}{ll}
\hline El profesor & El alumno \\
\hline Diseña actividades de enseñanza y aprendizaje & Realiza actividades de aprendizaje \\
Guía el aprendizaje & Construye su propio aprendizaje \\
Evalúa & Se autoevalúa \\
Elabora el diseño didáctico & Sabe que se espera de él \\
Diseña actividades sincrónicas y asincrónicas & Realiza actividades en grupo e individuales. \\
& Usa sus propios tiempos y ritmos de aprendizaje \\
Favorece las interacciones e interactividades & Realiza actividades en grupo e individuales mediadas por la tecnología \\
Usa foros de discusión & Usa sus propios tiempos y ritmos de aprendizajes \\
Elabora materiales de apoyo al aprendizaje & Cuenta con materiales de estudio \\
Da asesorías como una guía en el aprendizaje & Cuenta con apoyo real para sus dudas \\
Evalúa las diversas actividades & Se autoevalúa \\
\hline
\end{tabular}


tes en el diseño didáctico en modalidades alternativas, es el papel que desempeñan en el ambiente de aprendizaje, donde cohabitan la planeación didáctica, los materiales de apoyo al aprendizaje y los recursos didácticos tecnológicos los cuales actúan como mediadores entre el alumno, el profesor y el saber.

Un curso en una plataforma educativa consta de los siguientes espacios: a) Encuadre de la unidad de aprendizaje; b) Objetivos de aprendizaje; c) Contenidos temáticos ;d) Metodología didáctica; e) Cronograma de actividades; f) Materiales educativos de apoyo al aprendizaje; g) Recursos didácticos tecnológicos a utilizar; $\mathrm{y}$ h) Sistema de evaluación del aprendizaje.

El uso de la plataforma permite al maestro llevar el seguimiento de la participación de los grupos de trabajo, tanto en foros de discusión como en comunidades virtuales en forma síncrona o asíncrona, considerando la pertinencia y calidad de las aportaciones de sus integrantes. Asimismo, permite la interactividad del alumno con el material educativo y las interacciones continúas entre el maestro y el alumno y también entre estudiantes.

\section{Metodología de la investigación}

\section{Método}

La investigación es de tipo mixta, es decir, cuantitativa y cualitativa. Se aplica el enfoque cuantitativo con el objetivo de evaluar el rendimiento académico de los estudiantes cuando cuentan con material didáctico contextualizado en circuitos eléctricos e implementado en plataforma Moodle en la materia de Ecuaciones Diferenciales de la carrera de Ingeniería Electrónica, se elabora un diseño experimental con 2 grupos, uno de control y el otro experimental con aplicación sólo de un postest. La propuesta de estrategias didácticas se aplicó al grupo experimental, al mismo tiempo que el grupo control recibió sus clases tradicionales sobre el tema de ecuaciones diferenciales. Las clases fueron impartidas en salones con infraestructura física similar por dos maestros diferentes con la misma formación profesional. Para el enfoque cualitativo se utilizó la dinámica de grupo focal cuyo objetivo fue evaluar el impacto en los alumnos del uso de la plataforma Moodle.

\section{La población}

Estuvo conformada por los alumnos que cursaban la materia de Ecuaciones Diferenciales en este caso cinco grupos con un total de 91 estudiantes de las carreras de Ingenierías que ofrece el ITESCA.

\section{La muestra}

Se eligieron dos grupos de alumnos de los cinco de la población que cursaban la materia de Ecuaciones Diferenciales de la carrera de Ingeniería Electrónica. En este caso el grupo control y experimental estuvieron conformados por 14 y 16 alumnos respectivamente. El tipo de muestreo fue no probabilístico intencionado.

Características de los grupos. Los alumnos del grupo experimental cursaban las materias Ecuaciones Diferenciales y Circuitos Eléctricos por primera vez, mientras que algunos de los alumnos del grupo control las cursaban por segunda vez. El Instituto Tecnológico Superior de Cajeme introduce, como recurso tecnológico en el 2006, la plataforma moodle en el entorno virtual de aprendizaje (eitesca.edu.mx), en la cual se desarrolla el presente trabajo.

\section{Instrumentos de observación}

Los instrumentos que se utilizaron para recabar la información cuantitativa sobre el rendimiento académico de los estudiantes fueron mediante dos exámenes escritos uno sobre eventos contextualizados y el otro sobre eventos descontextualizados ambos de opción múltiple.

El instrumento que se utiliza para llevar a cabo la evaluación cualitativa consiste en cinco preguntas que se aplicaron al grupo experimental las cuales sirvieron para conocer su opinión respecto del uso de la plataforma Moodle en el curso.

\section{Realización del material didáctico de apoyo}

Para la realización del material de apoyo se usaron cuatro fases que son una adaptación de la propuesta de Camarena [34] de Diseños de cursos de matemáticas en línea.

\section{Fase de diseño tecnológico}

A las actividades se dedicaron cuatro horas presenciales y una hora virtual semanalmente durante las cinco primeras semanas del semestre enero-mayo 2013, se correlacionaron con los objetivos requeridos en el programa formativo, escolar y curricular de la materia de Ecuaciones Diferenciales de Ingeniería Electrónica. Cabe mencionar que las cinco primeras semanas del curso corresponden al tema de ecuaciones diferenciales de primer orden, en el que se contextualizaron las ecuaciones diferenciales en los circuitos eléctricos.

El recurso tecnológico utilizado para el desarrollo del curso es la plataforma Moodle, que tiene una gran variedad de configuraciones, en el Instituto es común usar la configuración semanal, que se emplea en este caso. El ITESCA cuenta con un equipo de técnicos encargados de disponer los materiales diseñados por los maestros, para cada uno de los cursos 
virtuales o semipresenciales ofrecidos en el instituto. Por tal razón se cuenta con plantillas que permiten la comunicación entre el maestro que diseña el material y el equipo técnico que lo pondrá en la plataforma.

De acuerdo al Modelo Didáctico de Cursos en Línea [34], son de especial importancia los materiales de apoyo al aprendizaje, los recursos didácticos tecnológicos y el ambiente de aprendizaje en el cual se desarrollan. Por tal razón es necesario, al hacer, uso de TIC en educación, el modelo educativo de la institución para la realización de la planeación del curso, el diseño de los materiales didácticos, los recursos tecnológicos, el proceso de evaluación para que el alumno desde el inicio tenga conocimiento global del papel que va a desempeñar en el desarrollo del curso.

La plataforma Moodle permite subir materiales, abrir foros de discusión, mapas conceptuales, asignaciones, glosarios, chats, exámenes en línea, talleres de ejercicios, trabajo en equipo, etcétera.

También se cuenta con diversos tipos de actividades de aprendizaje con uso de recursos tecnológicos.

1) El Glosario de conceptos tiene como objetivo que los estudiantes investiguen, en diversos libros, las definiciones indicadas de ecuaciones diferenciales y se familiaricen con las diferentes notaciones matemáticas de los conceptos, así como también, tengan presente los conceptos básicos de circuitos eléctricos y las leyes de Kirchhoff que estarán utilizando en el curso. Algunas características de éstas se especifican en el glosario, los alumnos pueden comentar las entradas de sus compañeros, al profesor le es permitido calificar las entradas mediante escalas determinadas por él; la presentación de este tiene diversos formatos y los términos se pueden agrupar en categorías. También es posible importar y exportar glosarios.

2) Foro. La plataforma Moodle ofrece diferentes tipos de foros, algunas de sus características son: a) Para uso general, cualquiera puede empezar un nuevo tema de debate; b) Cada participante plantea solo un tema y todos pueden contestar a cualquier tema; c) De debate único, es utilizado para debates cortos y aquí hay intercambio de ideas sobre un tema; d) De preguntas y respuestas, en este foro solo el profesor inicia el debate con una pregunta la cual será el tema de discusión en la que los alumnos participan con sus aportaciones pero no es posible que vean las respuestas de otros estudiantes. Las aportaciones pueden ser calificadas por el maestro; e) Es posible adjuntar la foto de cada participante; f) Las discusiones pueden verse anidadas, por rama, o presentar los mensajes más antiguos o los más nuevos primeros; g) Se pueden establecer fechas para las participaciones; y h) La participación puede ser por invitación del maestro u obligatoria, como lo determine el profesor.

3) Taller de ejercicios. Esta actividad se presenta como tarea en la cual se permite subir sólo un archivo donde se especifican los ejercicios a realizar, el tiempo que permanecerá disponible para el alumno y la fecha de entrega. Además, cuenta con un apartado en el cual el alumno puede subir a la plataforma el taller resuelto.

4) Examen en línea. Esta es una actividad en línea la cual permite al alumno realizar el examen y al terminar conocer el resultado obtenido.

5) Mapa Conceptual. Es una herramienta que permite la memorización, organización y representación de la información con el propósito de facilitar los procesos de aprendizaje, administración y planeación organizacional así como la toma de decisiones.

6) Examen presencial en clase.

7) Investigación en equipo de una aplicación de ecuaciones diferenciales en ingeniería electrónica, se hace la presentación al grupo usando herramientas tecnológicas.

8) Materiales básicos de apoyo tales como formularios, libros electrónicos recomendados, bibliografía, notas de clase, etc., estos materiales se encuentran en la plataforma virtual.

9) Calendario de actividades en el cual se especifican fechas de entrega y características de cada actividad, ya sea presencial o en línea.

\section{Fase de diseño didáctico}

Aquí se realiza la planeación del curso y se diseña el material didáctico de apoyo al estudiante, así como la inclusión de los recursos didácticos tecnológicos que permitirán que el alumno planee sus actividades de acuerdo a su estilo de trabajo. Se emplea la fase didáctica de la teoría educativa la Matemática en el Contexto de las Ciencias que fue mencionada en el marco teórico de este trabajo, esto con el fin de diseñar las actividades guía para los estudiantes y que puedan hacer uso de sus etapas para la resolución delos eventos contextualizados.

Los materiales de apoyo didáctico son: a) Glosario de conceptos de ecuaciones diferenciales; b) Glosario de conceptos de física; c) Elaboración de mapa conceptual; d) Elaboración de talleres de ejercicios no contextualizados; e) Elaboración de talleres de ejercicios contextualizados; f) Examen en línea; y g)Investigación de aplicaciones de ecuaciones diferenciales en el área de estudio

\section{Fase de colocación en plataforma}

Cuando el estudiante entra a la plataforma observa la página principal del curso de ecuaciones diferenciales donde observa los datos de la asignatura, calendario de actividades, notas de clase, etcétera. En la primera liga que puede entrar el 
estudiante: Datos asignatura se presenta la plantilla guía que visualiza el alumno desde el inicio del curso, el cual es sobre ecuaciones diferenciales como se había mencionado. Está elaborada por el profesor y un equipo experto en técnicas de enseñanza, además de ser avalado por la Academia de Matemáticas adscrita al Departamento de Ciencias Básicas del Instituto. Se presenta el calendario de actividades a desarrollar donde se especifica los temas, tipo de actividad, porcentaje de calificación, modo y fecha de entrega, así el alumno tiene la oportunidad de distribuir su tiempo y trabajar de acuerdo a sus posibilidades, como se menciona en el modelo de diseños de cursos en línea.

De acuerdo al modelo didáctico que menciona el uso de recursos didácticos tecnológicos, para este curso se han elegido foros de discusión, elaboración de mapa conceptual, aportación a glosario de conceptos de ecuaciones diferenciales y a conceptos de física, cuestionarios en línea, etcétera.

\section{Fase de inducción a los estudiantes}

De acuerdo a [34], se hace la inducción al alumno para el uso de la plataforma educativa a utilizar, ya que de la participación que se haga de la misma se obtienen evidencias de aprendizaje al registrarse las aportaciones en los grupos de trabajo colaborativo, en foros de discusión y demás actividades asignadas al alumno, así como también, la interactividad de los alumnos con los materiales educativos y la interacción existente entre maestro-alumno, alumno-alumno.

Para darse el alta en la plataforma ITESCA virtual el maestro envía la lista de los alumnos que tomarán el curso donde indica la matrícula, nombre y un correo electrónico del alumno a la Coordinación de Educación a Distancia y ellos generan el grupo.

Para el acceso al curso en plataforma entrar al sitio http:// www.e-itesca.edu.mx, en el campo Nombre de usuario se coloca la matrícula, en el campo Contraseña se coloca el CIP (Clave de Identificación Personal) que es proporcionado por el Departamento de Control Escolar al alumno el día de su inscripción en el instituto.

Una vez que se haya entrado, aparece el listado de todos los cursos del alumno en esta modalidad. Se selecciona el curso de ecuaciones diferenciales y aparece la página principal en la que visualizan los elementos que constituyen el curso y se les invita a que entren en cada una de las pestañas para que aprendan a navegar dentro de la plataforma.

Aquí el alumno puede consultar qué es, en qué consiste, cómo se hace y la plantilla guía de realización y entrega de: a) Resumen; b) Ensayo; c) Síntesis; d) Comparación; e) Mapa conceptual; f) Línea de tiempo; g) Cuadro sinóptico; y h) Ante- proyecto de investigación y construcción de apoyo.

Para interactuar con el contenido del curso en plataforma, entra en el bloque de Actividades, aquí aparecen todas las actividades programadas para el curso como los cuestionarios, foros, glosarios, recursos, tareas, etc. Se les indica a los alumnos cómo navegar en cada uno:

1. Cuestionario. Se selecciona el cuestionario a realizar, aparece la información, el número de intentos, el tiempo para realizarlo. Ya que se realiza se guarda y envía para su calificación.

2. Foro. Permite la interacción de los compañeros y con el profesor sobre un tema elegido. Se selecciona el foro y aparece el tema asignado por el profesor; para participar en él se elige responder, se hace la aportación y si se desea se puede adjuntar un archivo, se envía al foro respondiendo al maestro o a algún compañero en particular.

3. Glosario. Contiene los conceptos necesarios en el desarrollo del curso. Se elige en el que se desee hacer una aportación y en la sección de vista normal aparece el listado de términos, para hacer una aportación se da clic en agregar entrada se escribe el concepto, se envía al glosario, que puede ser visto por todos los compañeros.

4. Recursos. Se les indica que en Recursos ellos pueden tener acceso a cada uno de los materiales de apoyo al curso como son: a) El Libro de ecuaciones diferenciales en línea; b) Formularios de leyes de los exponentes y exponenciales; c) Formularios de la transformada de Laplace; y d) Notas de clase elaboradas por el maestro, etc.

5. Tarea. Se selecciona la tarea de la página principal del curso y aparece la descripción e información relacionada con la misma.

En el bloque de Administración se encuentra cómo visualizar las calificaciones, realizar el perfil y revisar los eventos próximos.

Calificaciones. Al ingresar a la liga de calificaciones aparece la lista de participantes del curso, así como todas las actividades evaluables con información, tales como la actividad a evaluar, calificación obtenida, rango de la calificación, porcentaje obtenido y la recomendación del maestro.

Perfil. Aquí se proporciona la información personal tal como nombre, dirección de correo electrónico, descripción personal, etc. También es posible modificar información adicional.

Bloque de eventos próximos. Presenta el acceso a las actividades a realizarse en fechas próximas; se puede visualizar el calendario de eventos próximos, es posible usarse como agenda personal en diferentes formatos. 


\section{Resultados}

\subsection{Resultados de la evaluación cuantitativa}

Para el análisis estadístico y la obtención de tablas de la información, se utilizó el paquete computacional SPSS versión 17.0, para las distintas variables independientes que se analizaron en el estudio, siendo la variable dependiente el rendimiento académico.

Las variables independientes analizadas son: a) Examen de conocimientos; b) Examen de eventos contextualizados; y c) Promedio.

Se hace un análisis comparativo entre cada una de las calificaciones obtenidas en los exámenes, para determinar el rendimiento académico de los grupos.

Los exámenes se aplicaron a un total de 30 alumnos inscritos en la materia de Ecuaciones Diferenciales de la carrera de Ingeniería Electrónica del Instituto Tecnológico Superior de Cajeme; los cuales se dividieron en 16 alumnos del grupo control y 14 del grupo experimental.

Las características de las variables son: Tipo de medición: a) Nominal; b) Reactivos de opción múltiple; c) Una sola respuesta; y d) Respuestas pre codificadas.

Con el fin de determinar las diferencias entre las calificaciones obtenidas entre grupo experimental y el grupo de control se realizaron con el paquete SPSS pruebas $t$ de student para dos

Tabla 1. Porcentaje de aprobación y reprobación según el tipo de examen.

\begin{tabular}{lcccc}
\hline \multirow{2}{*}{ Examen } & \multicolumn{2}{c}{ Grupo experimental } & \multicolumn{2}{c}{ Grupo control } \\
\hline & Aprobados & No aprobados & Aprobados & No aprobados \\
Conocimiento & $71 \%$ & $29 \%$ & $38 \%$ & $62 \%$ \\
Contextualizado & $50 \%$ & $50 \%$ & $38 \%$ & $62 \%$ \\
\hline
\end{tabular}

Tabla 2. Media de los grupos por tipo de examen.

\begin{tabular}{lllcc}
\hline Examen & Grupo & $\mathrm{N}$ & Media & $\begin{array}{c}\text { Desviación } \\
\text { estándar }\end{array}$ \\
\hline \multirow{2}{*}{ Conocimiento } & Experimental & 14 & 76.07 & 3.523 \\
& Control & 16 & 43.75 & 9.270 \\
Contextualizado & Experimental & 14 & 61.67 & 7.102 \\
& Control & 16 & 62.50 & 5.284 \\
\hline
\end{tabular}

Tabla 3. Media de los grupos.

\begin{tabular}{lcccc}
\hline Grupo & $\mathrm{N}$ & Media & $\begin{array}{c}\text { Desviación } \\
\text { estándar }\end{array}$ & $\begin{array}{c}\text { Error } \\
\text { estándar }\end{array}$ \\
\hline Experimental & 14 & 68.93 & 13.506 & 13.506 \\
Control & 16 & 53.13 & 23.425 & 23.425 \\
\hline
\end{tabular}

Tabla 4. Comparación de medias sobre la variable dependiente promedio.

\begin{tabular}{lcccccc}
\hline $\begin{array}{l}\text { Variable dependiente } \\
\text { promedio }\end{array}$ & F & Sig & t & gl & $\begin{array}{c}\text { Diferencia de } \\
\text { medias }\end{array}$ \\
\hline & 4.528 & 0.042 & 2.219 & 28 & 15.804 \\
\hline
\end{tabular}

muestras independientes, al total de 30 alumnos evaluados. A continuación, se hacen los análisis estadísticos de las tablas que se obtuvieron de las distintas variables independientes que se consideraron en esta investigación. En la Tabla 1 se muestra los porcentajes de aprobación y reprobación según el tipo de examen.

En la Tabla 2 se muestran las medias de los grupos obtenidas en cada uno de los exámenes, donde se puede apreciar una diferencia considerable entre ellos respecto al examen de conocimientos, siendo a favor del grupo experimental, mientras que en el contextualizado no se aprecia diferencia, lo cual pudo haber afectado la elección de los grupos, donde el grupo experimental cursaba por primera vez los cursos de Ecuaciones Diferenciales y Circuitos Eléctricos 1 y algunos alumnos del grupo control los cursaba por segunda ocasión.

En las tablas 3 y 4 se muestran los resultados obtenidos de la variable dependiente promedio de los dos exámenes donde se muestra la media de los grupos y la comparación de medias, donde se observa que entre los dos grupos analizados el experimental y el de control, sí existen diferencias significativas $(\mathrm{p}=.042)$, es decir, el rendimiento académico del grupo donde se aplicó el tratamiento si mejoró respecto al grupo de control.

\subsection{Resultados de la evaluación cualitativa}

Los resultados de la evaluación cualitativa sobre el uso de la plataforma Moodle fueron generados a través de la dinámica de grupos focales con los 12 alumnos que conformaron el grupo 
experimental y tres maestros, los 2 que atendieron al grupo control y experimental y uno adicional que participó como moderador y facilitador de las discusiones. La dinámica se desarrolló en una sesión de 90 minutos donde se abordaron algunas preguntas elaboradas previamente.

Específicamente se plantearon las siguientes preguntas:

1. ¿Qué opinan sobre el uso de la Plataforma Moodle en la enseñanza del curso de ecuaciones diferenciales?

Uno de los doce alumnos expreso que para él la matemática no debe ser virtual, pues considera que se entienden mejor los procedimientos en el pizarrón. En general los restantes expresaron que de entrada la plataforma Moodle no fue de su agrado, pero con el paso del tiempo esa percepción les fue cambiando pues vieron la importancia de la misma. También se comentó que no siempre tienen acceso a internet y no saben administrar, aún, su tiempo.

2. ¿Qué opinan de la comunicación alumno-maestro y alumnoalumno en la plataforma?

Parece haber acuerdo en que la comunicación alumno-maestro, alumno-alumno fue buena o muy buena y resaltan la importancia del uso del foro, ya que como se quedan escritos los comentarios de quienes participan, pueden entender mejor algún concepto porque está escrito y descrito en el foro, asimismo, mencionan que podían comprender mejor algún tema con ayuda de un amigo o del maestro; la participación continúa en el foro permite el diálogo escrito para dudas que le surjan a algún participante. Como es sabido en esta modalidad educativa la ventaja es que el estudiante no está solo para la construcción del conocimiento [35].

3. ¿Qué opinan sobre la utilización de: a) Foros de discusión, b) Mapas Conceptuales, c) Glosarios, d) Subir tareas y e) Editor de ecuaciones, etcétera?

Parece haber consenso en que se dificultó el uso de la plataforma Moodle al manejar el editor de ecuaciones ya que algunos no lo conocían y por las características propias del editor pues editar ecuaciones les resultaba laborioso, además se resalta la importancia del uso de glosarios y mapas conceptuales ya que con esto se organizan más las ideas y se comprenden mejor los temas.

4. ¿Cuál crees que es la aportación brindada a tu aprendizaje con el uso de la Plataforma Moodle?

Un alumno expreso que en lo personal ya no dependía tanto del maestro ya que podía construir su propio conocimiento, otros mencionaron que aprendieron a organizar el tiempo ya que tenían con anticipación que subir las tareas. En general la percepción es que se tiene una mejor organización del tiempo y se desarrolla un aprendizaje autónomo.
5. ¿Qué opinan sobre si el uso de la plataforma eleva el interés y la motivación en ustedes?

Algunos muestran tener un interés por aprender más sobre la tecnología refiriéndose a la plataforma Moodle y por ser simplemente un curso diferente al de clase solo expositiva por parte del maestro. Algunos otros muestran mejora en la motivación la cual adjudican a un mejor entendimiento de las cosas, se hace referencia también a un cambio de paradigma de la matemática de considerarla de hacer puros ejercicios a una donde ya le ven una utilidad "matemática contextualizada", otros expresan que con solo trabajar con la computadora el trabajo va a ser más dinámico y motivacional.

\section{Conclusiones}

El objetivo del presente trabajo de investigación fue por un lado estudiar la relación entre el indicador de rendimiento académico (calificación del tema "ecuaciones diferenciales de primer orden" del curso de Ecuaciones Diferenciales) y el factor del uso de material didáctico contextualizado en circuitos eléctricos e implementado en plataforma Moodle, en una muestra de dos grupos de estudiantes del Instituto Tecnológico Superior de Cajeme y por otro lado cual es la percepción de los estudiantes de trabajar con la plataforma Moodle respecto al proceso de enseñanza y aprendizaje.

Se aplicaron dos exámenes, uno fue el examen de conocimientos de resolución de ecuaciones diferenciales ordinarias de primer orden y el otro un examen de resolución de eventos contextualizados en circuitos eléctricos.

Basado en el análisis estadístico realizado en la sección anterior se puede observar que:

1. Respecto al examen de conocimientos, se concluye que los alumnos del grupo experimental, que tomaron el curso de ecuaciones diferenciales con el material didáctico de apoyo e implementado en plataforma Moodle, mejoró su rendimiento académico con respecto a los alumnos que llevaron el curso en forma tradicional.

2. En lo referente al examen de eventos contextualizados el rendimiento académico del grupo control, el cual llevó sus clases en forma tradicional fue ligeramente mejor que el grupo experimental.

3. Respecto a la comparación de las medias de ambos exámenes se concluyó que existen diferencias significativas en el rendimiento académico a favor del grupo que recibió el tratamiento.

Una variante que pudo haber afectado los resultados del punto 2 es que los alumnos del grupo experimental cursaban por primera vez la materia de ecuaciones diferenciales y circuitos 
eléctricos 1 y en el grupo control algunos alumnos ya habían cursado con anterioridad las materias mencionadas.

Por otro lado, se pudo observar que los alumnos del grupo experimental se mostraron más interesados en la materia debido a la dinámica del curso que fue más flexible e interactiva. El sentido de responsabilidad de los alumnos se vio reforzado al ellos determinar, en algunas de las actividades propuestas, sus tiempos de trabajo y entregas. El investigar los temas antes de cada clase y participar en la elaboración de los glosarios los motivó en el autoaprendizaje.

Con base en lo anterior se puede concluir que:

1. El profesor puede diseñar adecuadamente actividades didácticas con el uso de la plataforma Moodle que mejoren el rendimiento académico de los estudiantes en matemáticas.

2. La plataforma Moodle puede desarrollar en los estudiantes la colaboración, la autonomía y autorregulación del aprendizaje.

Como un punto a considerar para la mejora del uso de la plataforma en las clases de matemáticas habría que atender la opinión expresada por los alumnos respecto a la dificultad del uso de ésta cuando utilizan el editor de ecuaciones, es decir, mejorar la edición de expresiones matemáticas para eficientar la entrega en tiempo y forma de actividades solicitadas por el maestro.

Un estudio similar que coincide con el presente trabajo fue referente al mejoramiento del rendimiento académico con el uso de TIC y lo presentó Amelii [36], se realizó en la Universidad Central de Venezuela durante los semestres académicos 2005-02, 2006-01, 2006-02, 2007-01 y 2007-02 con los estudiantes que cursan la asignatura Matemática II en la Escuela de Economía. El objetivo de la investigación fue determinar la efectividad de las estrategias didácticas diseñadas en un curso en línea desarrollado para asesorar a distancia a los estudiantes repitientes y en régimen de permanencia en la Asignatura mencionada. Obteniendo como resultado:

En líneas generales, los resultados de este estudio mostraron que el rendimiento en la asignatura mejoró notablemente, los estudiantes manifestaron una gran satisfacción con la metodología empleada lo cual permitió mejorar su actitud ante la asignatura y contribuyó a disminuir los niveles de repitencia.

Sin embargo, existen estudios que difieren a los resultados del presente trabajo.

En [37], se presenta una investigación sobre la evaluación del impacto de la plataforma tecnológica denominada Sistema de
Apoyo a la Educación con Tecnología de Internet (SAETI). El estudio se realizó a 12 de 28 grupos que cursaron la materia Educación Tecnológica que se imparte en diferentes carreras de licenciatura ofrecidas en el Instituto Tecnológico de Sonora (ITSON). El propósito de este trabajo que se realizó el semestre enero-mayo de 2006, fue el evaluar, por un lado, a través del enfoque cuantitativo, la efectividad de la plataforma en el rendimiento académico de los alumnos y, por otro, determinar cualitativamente la utilidad de la misma desde la visión de los alumnos, profesores y personal que participó en su desarrollo.

Los resultados obtenidos indican que no se obtuvieron diferencias significativas en el aprovechamiento de los alumnos entre los grupos que usaron la plataforma SAETI respecto a los que no la usaron; sin embargo, cualitativamente se obtuvieron resultados satisfactorios de su utilización tanto por parte de los alumnos como de los profesores, los cuales coincidieron en que ayuda a promover el autoestudio, la comunicación alumno-maestro, a la mejor organización durante el desarrollo del curso. Además, de comentar su fácil manejo, acceso rápido y en general que ayuda en el aprendizaje de los alumnos.

\section{Referencias}

[1] P. Camarena, El currículo de las matemáticas en ingeniería, Memorias de las Mesas redondas sobre definición de líneas de investigación en el Instituto Politécnico Nacional, México, 1984.

[2] D. Ausubel, Psicología educativa: Un punto de vista cognoscitivo, México, Trillas, 1976.

[3] P. Camarena, Teoría de la Matemática en el Contexto de las Ciencias, Actas del III Coloquio Internacional sobre Enseñanza de las Matemáticas, Conferencia Magistral, pp. 83-107, Lima, Perú, Pontificia Universidad Católica del Perú, 2008. Disponible en http://irem.pucp.edu.pe/162/iii-coloquiointernacional-sobre-ensenanza-de-las-matematicas

[4] P. Camarena, "A treinta años de la teoría educativa Matemática en el Contexto de las Ciencias," Revista Innovación Educativa, vol. 13, núm. 62, pp. 17-44, 2013.

[5] C. R. Muro, "Análisis del conocimiento del estudiante relativo al campo conceptual de la serie de Fourier en el contexto de un fenómeno de transferencia de masa," Tesis Doctoral, Instituto Politécnico Nacional, México, 2004, Disponible en http://repositoriodigital.ipn.mx/handle/123456789/11657

[6] C. Olazábal, "Categorías en la traducción del lenguaje natural al algebraico de la matemática en contexto," Tesis de Maestría, Centro de Investigación en Ciencia Aplicada y Tecnología Avanzada, México, 2005.

[7] E. Trejo, "La Ecuación Diferencial en el Contexto de las Reacciones Químicas de primer Orden," Tesis en Maestría, Universidad Autónoma del Estado de Hidalgo, México, 2005.

[8] P. DePavia, "Desarrollo de habilidades del pensamiento para la matemática en el contexto de las ciencias," Tesis de Maestría, 
Centro de Investigación en Ciencia Aplicada y Tecnología Avanzada, México, 2006.

[9] T. M. Sauza, "Una propuesta didáctica del análisis matemático en el contexto de la ingeniería de control," Tesis de Maestría, Universidad Autónoma del Estado de Hidalgo, México, 2006.

[10] P. Y. Alvarado, "Análisis del significado de la solución de las ecuaciones diferenciales lineales en la volatización de compuestos orgánicos," Tesis de Maestría, Universidad Autónoma del Estado de Hidalgo, México, 2008.

[11] B. J. Rojas, "Aplicación de los campos de Galois en el contexto de la corrección y detección de errores en comunicaciones basadas en los códigos $\mathrm{BCH}$, con un enfoque didáctico," Tesis de Maestría, Ciudad de México: Instituto Politécnico Nacional, 2008.

[12] H. J. Accostupa, "Propuesta didáctica para las funciones sinusoidales de la forma $\mathrm{f}(\mathrm{x})=\mathrm{A}+\mathrm{BSen}(\mathrm{Cx}+\mathrm{D})$ en el contexto de los circuitos eléctricos del área de la Ingeniería," Tesis de Magíster, Pontificia Universidad Católica del Perú, 2009.

[13] F. V. Neira, "Modelación de problemas contextualizados usando sistemas de ecuaciones lineales con dos variables: basado en el enfoque de la Matemática en el Contexto de las Ciencias," Tesis de Magíster, Pontificia Universidad Católica del Perú, 2012.

[14] P. Camarena, A. I. P. Flores, "La interdisciplinariedad: nivel superior. Colección: Experiencias de investigación. Tomo III," en Procesos de enseñanza y aprendizaje: estudios en el ámbito de la educación media superior y superior. Coordinadores: Gutiérrez R. D., Ceniceros D. C., Monárrez V. H. pp. 150167. Red Durango de Investigadores Educativos AC, México: REDIE, 2012.

[15] P. Camarena, "Diseño de un curso de ecuaciones diferenciales en el contexto de los circuitos eléctricos," Tesis de Maestría, Centro de Investigación en Ciencia Aplicada y Tecnología Avanzada, México, 1987.

[16] L. Meza, G. Garita, L. Villalobos,"Estrategias Didácticas para Desarrollar Procesos de Enseñanza y Aprendizaje de la Matemática Asistidos por Computadora." Memorias del II Congreso Internacional de Matemática Asistida por Computadora, vol. 1, núm. 1, pp. 84-96. 2001.

[17] E. Vilchez, "Sistemas expertos para la enseñanza y el aprendizaje de la matemática en la educación superior," Cuadernos de investigación y formación en educación matemática, núm. 3, pp. 45-67, 2007.

[18] B. Kolman, Álgebra lineal con aplicaciones y Matlab. México: Pearson, 1999.

[19] Commision on Higher Education MSA: Framework for Outcomes Assessment. Middel State Association, 1995.

[20] S. Rodríguez, E. Fita, M. Torrado,"El rendimiento académico en la transición secundaria-universidad," Revista de Educación, Temas actuales de enseñanza, ným. 334, pp. 391-414, 2004.

[21] M. Moreno, C. Azcárate, "Concepciones y creencias de los profesores universitarios de matemáticas acerca de las ecuaciones diferenciales," Enseñanza de las ciencias, 265-280, 2003

[22] R. Cantoral, "Enseñanza de las matemáticas en la educación superior," Revista electrónica sinéctica, pp. 3-27. 2001.

[23] A. Pérez, J. Ramón \& J. Sánchez, Análisis exploratorio de las variables que condicionan elrendimiento académico, Sevilla: Universidad Pablo de Olavide. 2000.

[24] A. Vélez, C. N. Roa,"Factores asociados al rendimiento académico en estudiantes deMedicina," PSIC Educación Médica, vol. 8, núm. 2, pp. 74-82, 2005. Recuperado de http:/ /scielo.isciii.es/pdf/edu/v8n2/original1.pdf

[25] G. M. Garbanzo, "Factores asociados al rendimiento académico en estudiantes universitarios desde el nivel socioeconómico: Un estudio en la Universidad de Costa Rica," Revista electrónica educare, vol. 17, núm. 3, 2013.

[26] P. Camarena, "La enseñanza de las matemáticas en el contexto de la ingeniería", Memorias del XXVIII Congreso Nacional de la Sociedad Matemática Mexicana, 1995, pp. 28-34.

[27] P. Camarena, Las funciones generalizadas en ingeniería, construcción de una alternativa didáctica, México, 2001a, ANUIES.

[28] P. Camarena, "La matemática en el contexto de las ciencias: las competencias profesionales," Reporte de investigación, México, 2005, ESIME-IPN.

[29] P. Camarena, "La matemática formal en la modelación matemática," Reporte de investigación, México, 2007, ESIMEIPN.

[30] P. Camarena, Curso de análisis de Fourier en el contexto del análisis de señales eléctricas, IPN: México. 1993.

[31] P. Camarena, "Reporte del proyecto de investigación titulado. Etapas de las matemáticas en el contexto de la ingeniería,". México: ESIME-IPN, 2000.

[32] M.A. Hernández, "Las ecuaciones diferenciales ordinarias de primer y segundo orden en el contexto del movimiento uniforme," Tesis de Maestría, Instituto Politécnico Nacional, México. 2009.

[33] E. Trejo, P. Camarena, N. Trejo, "Las matemáticas en la formación de un ingeniero: la matemática en contexto como propuesta metodológica," Revista de Docencia Universitaria, núm. 11, pp. 397-424. 2013.

[34] P. Camarena, Diseño de cursos en línea, Jalisco, México, 2010.

[35] P. Camarena, "Guía para el diseño didáctico en modalidades alternativas," Reporte de investigación, México, 2006, DINME-SA-IPN.

[36] R. Amelii, "Asesoramiento Académico en Línea para Estudiantes Repitientes en el área de Matemática," Docencia Universitaria, núm 1, 2011.

[37] O. Cuevas, R. I. García, I. R. Cruz, "Evaluación del impacto de una plataforma para la gestión del aprendizaje utilizada en cursos presenciales en el Instituto Tecnológico de Sonora," Revista Mexicana de investigación Educativa, vol. 13, núm. 39, 2008. 
